\title{
A Review of "Cost Effectiveness Modelling for Health Technology Assessment: A Practical Course"
}

\author{
Ahmed Abdulla $^{1} \cdot$ Angela Stainthorpe $^{1}$
}

Published online: 7 April 2016

(c) Springer International Publishing Switzerland 2016

This book by Edlin and colleagues [1] is a timely addition to the dearth of practical guides to cost-effectiveness modelling. The book focuses on the applied quantitative methods that meet the methodological standards set by organisations responsible for health technology assessment (HTA) such as the National Institute for Health and Care Excellence (NICE) in the UK and the Canadian Agency for Drugs and Technologies in Health (CADTH). Each chapter of this course breaks down and presents a key aspect of decision analytic modelling whilst providing well-defined interpretations of how the range of methods inform the decision-making process.

The subject material is spread across 13 chapters; the chapters are fairly self-contained and could be read independently if required. The book's key asset is the accompanying practical exercise workbook. The methodological approach employed in each chapter is built upon with a clear, step-by-step, empirical implementation that is easy to follow. The book's associated website (http://medhealth. leeds.ac.uk/costeffectivenessmodelling) provides readers with worksheets and their practical solutions. To complete the exercises, the reader needs a basic working knowledge of Microsoft Excel.

Following a general overview of the methods of economic evaluation in Chapter 1, there is a helpful

Ahmed Abdulla

ahmed.abdulla@liverpool.ac.uk

Angela Stainthorpe

angela.stainthorpe@liverpool.ac.uk

1 Liverpool Reviews and Implementation Group (LRiG), University of Liverpool, Second Floor, Whelan Building, The Quadrangle, Brownlow Hill, Liverpool L69 3GB, England introduction outlining how to source evidence to be used in cost-effectiveness modelling in Chapter 2. Identification of robust evidence is an important stage in the development of economic evaluations for HTA and this step is crucial before defining a relevant model structure and identifying appropriate parameter values. Guides to cost-effectiveness modelling do not generally include this kind of advice, even though it is a primary requirement of any good model, so the reader will welcome its inclusion here. Using key concepts, Chapters 3 and 5 provide an introduction to building decision trees and Markov models. Chapters 9 and 10 further develop these two chapters by equipping the reader with the basic skills to build Markov models in Excel and make the models probabilistic. The exercises for these chapters (available online) are well thought out and focus on the key technical aspects that give cost-effectiveness models their distinct characteristics. Chapter 4 deals with the uncertainty inherent in the methods and outputs of cost-effectiveness analyses. However, some topics introduced in this section such as sensitivity analysis and model outputs may be more suited to later chapters.

The authors focus on probability distributions for effectiveness parameters, and cost and utility data in Chapters 6 and 7, respectively. These chapters clearly define different distributions and thoroughly explain their implementation in cost-effectiveness models. Chapter 8 considers the effect of correlation between model parameters and the use of Cholesky Decomposition to integrate this into probabilistic analyses. The reader needs a basic appreciation of medical statistics to fully benefit from the material and exercises related to Chapters 6, 7 and 8 . Chapter 11 deals with the output of probabilistic cost-effectiveness results using the standard methods that are accepted by HTA bodies. The final chapters of this 
practical guide, Chapters 12 and 13, describe the techniques that decision makers or payers use when considering the reimbursement of technologies. Whilst these chapters comprehensively address the main issues, they are concise enough to not make the reader lose interest.

As health economic modellers and recent students of cost-effectiveness modelling, we found this book to be a good alternative to current modelling guides, and we were particularly pleased with its practical focus. The Excel worksheets available online are clear and easy to understand, and the accompanying step-by-step exercise workbook is very helpful.

There is no substitute for learning by doing, and this book meets this need in a way that most similar publications do not. However, the content is not comprehensive and it does miss some important basic elements. For instance, the book omits any reference to budget impact modelling and it does not cover partitioned survival modelling, which is commonly used in cost-effectiveness modelling in oncology.

The chapters, for the most part, stand alone and there are certainly advantages to using the book as a reference. However, the book's title refers to it as a course and we feel that, to be a proper course, there needs to be a bit more flow between subject matter. In future editions we would recommend the inclusion of additional case-study exercises, perhaps following the development of a cost-effectiveness model for an HTA submission 'from scratch'. Linking these exercises to a narrative describing typical or example time frames, tasks and data sources-a sort of life story of a cost-effectiveness model-might also help to bring topics to life, especially for readers who are not currently working in HTA. We would also recommend a rearrangement of the contents of some of the chapters so that they more closely match the exercise workbook.
This book works as both a textbook and as a guide. Since the exercises are optional, it will appeal both to readers wishing to learn more about applied modelling methodology and to those wanting to understand what goes into a cost-effectiveness model without ever having to open an Excel file. The first half of the book gives good insight into some of the less technical features of cost-effectiveness modelling and should be fairly easy to follow for most people with some experience of the subject. The later chapters are more complex and readers do need to be familiar with basic cost-effectiveness modelling concepts and statistics to fully understand the text.

This book will be helpful to students and early-career modellers who are keen to develop their skills in costeffectiveness modelling. We would further recommend the text to professional health economists working outside of the UK and Canadian contexts who are aiming to widen their understanding of international approaches to cost-effectiveness modelling.

\section{Compliance with ethical standards}

Conflict of interest Ahmed Abdulla and Angela Stainthorpe declare that they have no conflict of interest.

\section{References}

1. Edlin R, McCabe C, Hulme C, Hall P, Wright J. Cost effectiveness modelling for health technology assessment: A practical course. Heidelberg: Adis: Springer Cham; 2015. 